\title{
THE OPTIMAL ROS-BASED SYMMETRIC PHASE-ONLY FILTER FOR FINGERPRINT VERIFICATION
}

\author{
Xin Shuai ${ }^{1}$, Chao Zhang ${ }^{1}$ and Pengwei Hao ${ }^{1,2}$ \\ ${ }^{1}$ State Key Laboratory of Machine Perception, Peking University, Beijing, 100871, China \\ ${ }^{2}$ Department of Computer Science, Queen Mary, University of London, E1 4NS, UK \\ Email: c.zhang@pku.edu.cn
}

\begin{abstract}
Symmetric phase-only filter (SPOF) has been widely applied to image registration and recognition, and has been proved efficient for fingerprint verification. Fingerprint images have a characteristic that the dominant information is concentrated in an elliptic frequency band of their ridges in low frequency domain. However, the existent fingerprint verification methods based on SPOF do not take this characteristic into account. To improve the performance of SPOF for fingerprint recognition, an appropriate region of support (ROS) can be used to set the least significant frequency region to zero. By means of theoretical and experimental analysis, we have found the optimal ROS for SPOF that achieves the best discrimination power among all the possible ROSs. Experiments show that our optimal ROS-based SPOF is more efficient than the method of band-limited SPOF (BLPOC).
\end{abstract}

Index Terms - symmetric phase-only filter, region of support, matched filters, biometrics, fingerprint identification

\section{INTRODUCTION}

A fingerprint is a pattern of ridges and furrows on the surface of a fingertip. Its uniqueness and stability make fingerprint verification the most popular biometric technique for personal identification. Typical approaches of automatic fingerprint matching in the literature can be roughly classified into two categories, i.e. minutiae-based and correlation-based $[1,2]$.

Most fingerprint identification systems employ minutiaebased approaches. In these approaches, minutiae points such as ridge endings and bifurcations are extracted from ridge lines, and line orientation or the number of lines between every pair of minutiae points is calculated. These features are used for fingerprint verification [2]. However, minutiae-based approaches depend heavily on the extraction of reliable minutiae features. It has been known that fingerprints verification is a very difficult task if only minutiae-based techniques are applied due to various skin conditions or poor quality of images [3].

Unlike minutiae-based methods, correlation-based approaches extract features directly from the raw image. More discriminatory features can be obtained than the minutiaebased features since a gray-level image contains much richer information. When the image quality is too poor to extract reliable minutiae, correlation-based methods may be the only choice. It has been proved that correlation-based technique is more effective for identifying poor quality fingerprints [3]. The symmetric phase-only filter (SPOF) method is a correlation-based technique which has been demonstrated to be effective for fingerprint verification [4]. However, the original SPOF method has no noise suppression ability since it is a unit-magnitude filter (all-pass filter) operating on the spectral phase of the input image, and this property leads to bad discrimination capacity. To address this problem, band-limited SPOF (BLSPOF, called "BLPOC" in [5]) has been defined. As known to all, a fingerprint image has a special structure in the frequency domain that contains a significant elliptic band, but BLSPOF has not figured out the desirable region of support (ROS) of SPOF for fingerprint verification, thus cannot obtain a desirable result.

In this paper, an optimal ROS-based SPOF for fingerprint verification is proposed. Firstly our discussion focuses on the correlation performance measure (SNR) to determine ROS. We show that different frequency components have different discrimination capabilities which are related to the spectral magnitude. Then an optimal ROS is selected from a table of discrimination capabilities of the ROS candidates. Finally, our approach to determine ROS for fingerprint verification is presented.

This paper is organized as follows: Section 2 gives the definition of POF and SPOF. Section 3 is about the selection of the optimal ROS of SPOF for fingerprint matching via theoretical and experimental analysis. Section 4 presents a set of experimental results. In Section 5, we end with some conclusions.

\section{PHASE-ONLY FILTER AND SYMMETRIC PHASE-ONLY FILTER}

The classical matched filter has the limitation that the output of the filter is primarily dependent on the energy of the image rather than on its spatial structures. Furthermore, the matched filter output around its maximum is so broad that the peak cannot be easily located. The phase-only filter (POF) was proposed to solve such problems $[4,5,6]$.

Consider two $N_{1} \times N_{2}$ images, $f\left(n_{1}, n_{2}\right)$ and $g\left(n_{1}, n_{2}\right)$. Let $F\left(k_{1}, k_{2}\right)$ and $G\left(k_{1}, k_{2}\right)$ be the $2 \mathrm{D}$ discrete Fourier transforms of the two images. In the definition of POF which uses the spectral phase of $g\left(n_{1}, n_{2}\right)$ as the filter's transfer function, the output is the 2D inverse discrete Fourier transform of $R_{P O F}\left(k_{1}, k_{2}\right)$ which is given by 


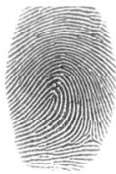

(a)

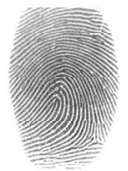

(b)

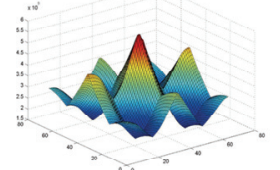

(c)

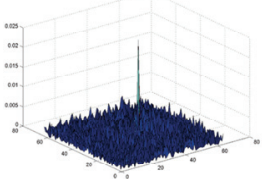

(d)
Fig. 1 Comparison between SPOF and classical matched filter. (a) and (b) are two identical fingerprint impressions, (c) SPOF between (a) and (b), (d) classical matched filter between (a) and (b).

$$
R_{P O F}\left(k_{1}, k_{2}\right)=F\left(k_{1}, k_{2}\right) \cdot \frac{G^{*}\left(k_{1}, k_{2}\right)}{\left|G\left(k_{1}, k_{2}\right)\right|}
$$

where $G^{*}\left(k_{1}, k_{2}\right)$ is the complex conjugate of $G\left(k_{1}, k_{2}\right)$.

A further improvement of POF can be achieved by extracting and correlating the phase of both $f\left(n_{1}, n_{2}\right)$ and $g\left(n_{1}, n_{2}\right)$. This is done by a nonlinear filter called SPOF with an output given by the 2D IDFT of the function

$$
R_{S P O F}\left(k_{1}, k_{2}\right)=\frac{F\left(k_{1}, k_{2}\right)}{\left|F\left(k_{1}, k_{2}\right)\right|} \cdot \frac{G^{*}\left(k_{1}, k_{2}\right)}{\left|G\left(k_{1}, k_{2}\right)\right|}
$$

We can observe that SPOF is taken as a POF operating on the image with the phase of $f\left(n_{1}, n_{2}\right)$ and unit-magnitude.

Since the spectral phase preserves the location of objects in the image but is insensitive to the image energy, a SPOF yields a much sharper peak than the classical matched filter when the two images are similar and a significantly lower peak when the two images are not similar. This property of SPOF is shown in Fig.1. Thus, the SPOF exhibits better discrimination capability.

Although SPOF can yield sharper correlation peak compared to a matched filter, it provides lower output SNR since a matched filter is designed to maximize the output SNR. As described before, SPOF is a filter operating on the phase of the input image with a magnitude of 1 at all frequencies. It does not suppress any noise in the signal. To improve the noise tolerance of the SPOF, we will find the optimal ROS for SPOF in the next section.

\section{THE OPTIMAL ROS FOR SPOF}

As explained in the above, SPOF is actually an all-pass filter for complete frequency range of the input image's spectral phase. We can observe from the frequency domain of a fingerprint image that significant information of the image is concentrated in an elliptic frequency band of its ridge lines, and the high frequency domain includes meaningless noise. Thus an all-pass filter that does not distinguish the meaningful information from noise will lead to a lower correlation peak.

One way to remove noise for a SPOF is to set some frequencies of the filter to zero [6]. The set of frequencies in which the filter magnitude is non-zero is called the region of support (ROS). BLSPOF method automatically detects the borders of ROS simply by calculating the mean values of the $k_{1}$-axis and $k_{2}$-axis projection of the spectral magnitude respectively, and eliminates the higher frequency components beyond the rectangle region. We believe that the ROS determination method proposed in BLSPOF does not give the optimal ROS thus does not achieve a desirable discrimination capability. In this section, a theoretical and experimental analysis will be presented to show how to find a better optimal ROS for SPOF.

\subsection{SNR analysis of SPOF with ROS}

Signal-to-noise ratio (SNR) is an important metric for designing correlation filters. It measures the noise sensitivity of the filters. To find an optimal ROS for SPOF, it is a natural way to find the region that maximizes the output SNR of the filter [6]. In order to obtain the expression of SNR, we switch back to $1 \mathrm{D}$ notation for simplicity.

Let $f_{i}(t)$ denote the input signal and $h(t)$ denote the impulse response of the correlation filter. We will consider the effects of additive noise $n(t)$ only for simplicity. Thus the problem is simplified as choosing between the two following hypotheses:

$$
\begin{aligned}
& H_{0}: f_{i}(t)=n(t) \\
& H_{1}: f_{i}(t)=f(t)+n(t)
\end{aligned}
$$

where $f(t)$ denote the transmitted signal. The noise $n(t)$ is modeled as a wide sense stationary (WSS) random process with zero mean and power spectral density (PSD) $P_{n}(f)$.

A desirable correlation filter should make the average maximal value $r_{\max }$ large (under hypothesis $H_{1}$ ) and make the average noise-included variance as small as possible. So the output SNR can be defined as

$$
S N R=\frac{\left|E\left\{r_{\text {max }} \mid H_{1}\right\}\right|^{2}}{\operatorname{var}\left\{r_{\text {max }}\right\}}
$$

Without loss of generality we may assume that $f_{i}(t)$ contains the un-shifted signal $f(t)$ and the noise, and thus the filter output achieves its maximal value at the origin, which is denoted as $r(0)$. Then the numerator of Eq.(4) can be simplified as

$$
\begin{aligned}
& \left|E\left\{r_{\max } \mid H_{1}\right\}\right|^{2}=\left|E\left\{r(0) \mid H_{1}\right\}\right|^{2} \\
& =\left|\int[f(t)+n(t)] h(-t) d t\right|^{2}=\left|\int F(f) H(f) d f\right|^{2}
\end{aligned}
$$

where $f(t)$ and $h(t)$ are assumed to be real. Since the noise $n(t)$ is WSS, we can express the denominator of Eq.(4) as

$$
\operatorname{var}\left\{r_{\max }\right\}=\int P_{n}(f)|H(f)|^{2} d f
$$

Using Eq.(5) and Eq.(6), SNR is obtained as follows:

$$
S N R=\frac{\left|\int F(f) H(f) d f\right|^{2}}{\int P_{n}(f)|H(f)|^{2} d f}
$$

Now we return to our task to find the optimal ROS for SPOF that maximizes the output SNR given in Eq.(7). Due to the nonlinearity of SPOF, we take it as a POF operating on an image with unit-magnitude (we have discussed in Section 2). Thus the problem is to find the optimal ROS for POF with unit-magnitude input.

Let $R$ denote the set of frequencies for which the filter magnitude is non-zero. Then the SNR is given as

$$
S N R_{R}=\frac{\left|\int_{R} F(f) H(f) d f\right|^{2}}{\int_{R} P_{n}(f)|H(f)|^{2} d f} \cong \Delta f \frac{\left[\sum_{k \in R} F_{k}\right]^{2}}{\sum_{k \in R} P_{k}}
$$

where $F_{k}$ and $P_{k}$ are samples of $|F(f)|$ and $P_{k}(f)$ sampled at uniform intervals of $\Delta f$. To determine the region that maxi- 
mizes the SNR in Eq.(8), we re-index the frequencies such that the ratio $F_{k} / P_{k}$ is sorted. Remember that the input is unit-magnitude, i.e. $\left|F_{k}\right| \equiv 1$, so the ratio sorting refers to sorting just $P_{k}$ as

$$
P_{1} \leq P_{2} \leq \cdots \leq P_{N}
$$

where $N$ is the total number of frequency samples. It is proved that the optimal $R$ includes all the frequencies with an index lower than $n$ if it includes frequency $n$ from the sorted list in Eq.(9). This means that the optimal $R$ includes $M$ frequency samples with the lowest noise PSDs. The problem is: How to determine $M$ to obtain the best performance of SPOF?

\subsection{Experimental analysis of SPOF with ROS}

Now the task turns to finding the frequencies with low noise PSD. How to measure the effect of the noise? An experiment which employs equal error rate (EER) as a criterion has been designed. EER is defined as the error rate where the false acceptance rate (FAR) and the false reject rate (FRR) are equal. When a frequency component mainly contains meaningful information for the discrimination task, it should show us some discrimination capability, and gives a low EER as a result. On the contrary, when a frequency component mainly contains noise, its result of EER should be around $50 \%$ (i.e. poor discrimination capability).

FVC 2002 DB1 which consists of 800 impressions provided by 100 fingers was used for our experimental analysis. For the FRR test, each impression of each finger is matched against all other impressions of the same finger. For the FAR test, the first impression of each finger is matched against the first impression of all other fingers. So there are totally 2800 genuine attempts and 4950 impostor attempts. Alignment has been done and common area has been extracted for each matching pair without any pre-processing. In all of our experiments, the FFT outputs are set to be 512 $\times 512$ points with the coordinate origin in the center. In order to extract the ROS composed of frequency samples, we use a mask of $512 \times 512$ with an annular area of value 1 and the other area to be 0 . Fig. 2 shows a mask operated on a spectrum. $\left(R_{\min }, R_{\max }\right)$ can be adjusted to form different masks. When multiplying the mask to amplitude spectrum of an image, the annular area with value 1 is extracted.

To analyze the frequency components, we perform matching tests with masks each with 10 points wide nonzero band. The masks are given by

$$
\left(R_{\min }, R_{\max }\right)_{i}=(10 i, 10 i+10), \quad i=0,1, \cdots 24
$$

and the results are illustrated in Fig.3.

Experiment results show that for the frequency bands higher than 90 points, the EERs are too high (higher than $30 \%$ ) to indicate any discrimination capability. We also present the mean value of magnitude sum of each frequency band in Fig.3. It can be observed that frequency components with good EER results also have significant spectral magnitude. The best EER results fall in bands of $(40,50),(50,60)$ and $(60,70)$, which are also the frequencies of the magnitude peaks. This is because these components include the frequency range of the fingerprint ridge lines. It implies that
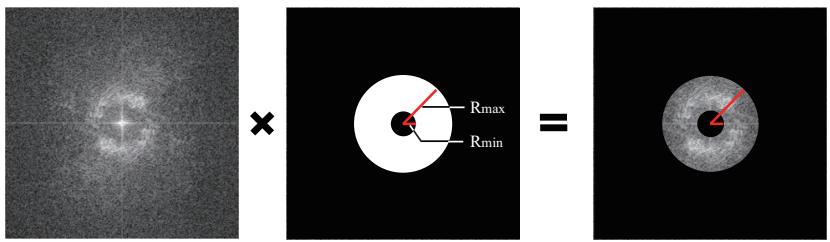

Fig. 2 Mask operated on a spectrum. (a) a frequency spectrum, (b) mask $\left(R_{\min }, R_{\max }\right)$, (c) result of mask operation.

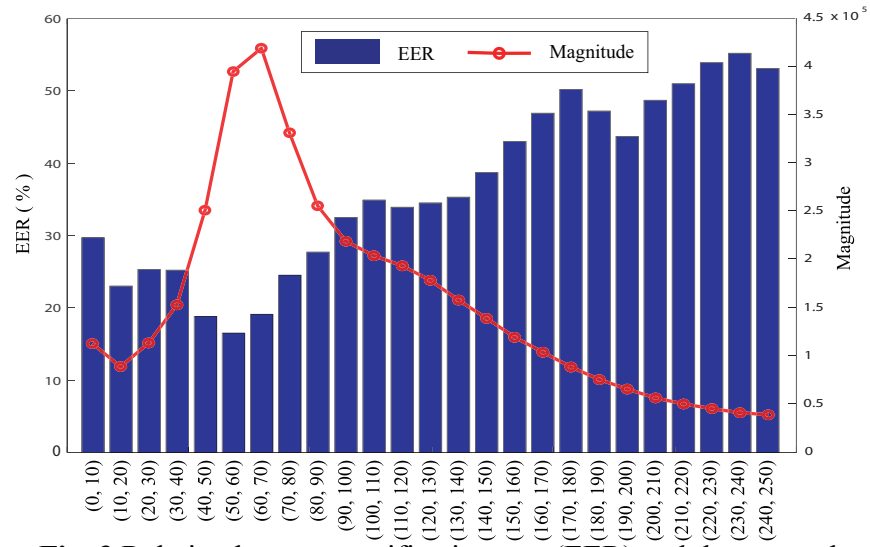

Fig. 3 Relation between verification rate (EER) and the spectral magnitude of different frequency range with an interval of 10 .

Table 1 Performance comparison for SPOF with different ROS

\begin{tabular}{r|rrrrr}
\hline EER(\%) & $\mathrm{R}_{\min }=0$ & 10 & 20 & 30 & 40 \\
\hline $\mathrm{R}_{\max }=70$ & 7.0 & 7.2 & 7.6 & 7.9 & 8.0 \\
80 & 6.2 & 6.2 & 6.4 & 6.2 & 6.6 \\
90 & 5.4 & 5.6 & 5.7 & 5.9 & 6.1 \\
100 & 5.7 & 5.9 & 6.0 & 6.0 & 6.2 \\
110 & 6.3 & 6.3 & 6.0 & 6.1 & 6.5 \\
120 & 7.0 & 6.9 & 6.4 & 6.3 & 6.9 \\
130 & 7.5 & 7.3 & 7.4 & 6.8 & 7.2 \\
140 & 8.9 & 8.4 & 7.8 & 7.7 & 7.8 \\
150 & 11.2 & 11.1 & 10.1 & 9.7 & 9.6 \\
\hline
\end{tabular}

we can determine ROS by detecting the peak of the magnitude sum of each frequency band.

Another experiment has been carried out to show how to select the optimal ROS that leads to the overall best EER. From the first experiment we learn that frequency band $(40,70)$ shows good discrimination capability among all frequency components, thus the optimal ROS should include these samples of frequency. We tested all the possible ROS and obtained the EER results given in Table 1. It can be clearly observed from Table 1 that the best result is achieved with the $\operatorname{ROS}(0,90)$. As the region changes, EER turns higher accordingly. So this region is the optimal ROS that we are looking for.

Actually, the frequency components of band $(0,90)$ can be classified into two parts. Band $(40,90)$ is the range corresponding to the fingerprint ridge lines. This part contains the information of ridge structure such as the flow directions. It is the most important information of which a correlation filter makes use, and it gives the lowest EER. The other part is the frequency ranges lower than 40 . This range mainly 


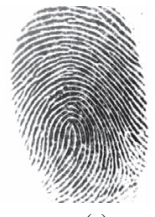

(a)

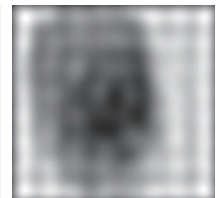

(b)

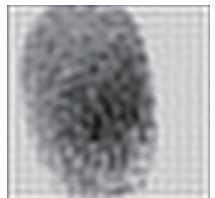

(c)

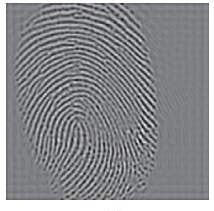

(d)
Fig. 4 2D IDFTs of different frequency range.

(a) the original image, (b), (c) and (d) are 2D IDFTs of frequency range $(0,10),(0,30)$ and $(40,70)$, respectively.

includes the information of the changes of gray-level and shows the discrimination capability of low frequencies. Fig.4 shows the 2D IDFTs of different frequency ranges.

\subsection{Method of optimal ROS determination}

As we know, the optimal ROS for SPOF is composed of the frequency ranges of the fingerprint ridge lines and the lower frequency components. Therefore, the optimal ROS can be determined by the outer boundary of the frequency range corresponding to the ridge lines. To detect this boundary, we use radial spectral magnitude distribution function $D(r)$ defined as follows [7]:

$$
D(r)=\frac{1}{N\left\{C_{r}\right\}} \sum_{\left(k_{1}, k_{2}\right) \in C_{r}}\left|F\left(k_{1}, k_{2}\right)\right|
$$

where $C_{r}$ is the set of the pixels that satisfy the function $k_{1}{ }^{2}+k_{2}^{2}=r^{2} . N\left\{C_{r}\right\}$ is the number of the elements of the set $C_{r}$. Besides the sharp peak at the origin, $D(r)$ has another crest corresponding to the dominant frequency of the fingerprints.

Thus the optimal ROS of an input image $f\left(n_{1}, n_{2}\right)$ can be determined by an algorithm consists of the following steps:

1. Compute the 2D DFT $F\left(k_{1}, k_{2}\right)$ of $f\left(n_{1}, n_{2}\right)$,

2. Compute $D(r)$ of $F\left(k_{1}, k_{2}\right)$,

3. Smooth $D(r)$ by proper Gaussian filter,

4. Detect the two local maximum values $V_{1}^{\max }$ and $V_{2}^{\max }$ and the local minimum value $V^{\text {min }}$ between the two maximums of the smoothed function,

5. Set the border of the optimal ROS as $2 V_{2}^{\max }-V^{\min }$.

\section{EXPERIMENTAL RESULTS}

Our experiment has been conducted on the database FVC 2002 DB1, which we have introduced in Section 3.2. Equal error rate (EER) is used for the performance evaluation. Raw images without any preprocessing were used in our experiment. We carried out experiments by original SPOF, BLSPOF and SPOF with optimal ROS in order to compare the performance of these correlation filters. Before the operation of the filters, we aligned each pair of images and extracted their common regions.

Three experiment results are shown in Fig.5. EER result of experiments using original SPOF, BLSPOF and SPOF with optimal ROS are $11.84 \%, 7.57 \%$ and $5.43 \%$ respectively. It can be seen that the proposed optimal ROS achieved the best performance among the three filters.

\section{CONCLUSIONS}

This paper presented the optimal ROS-based SPOF for fin-

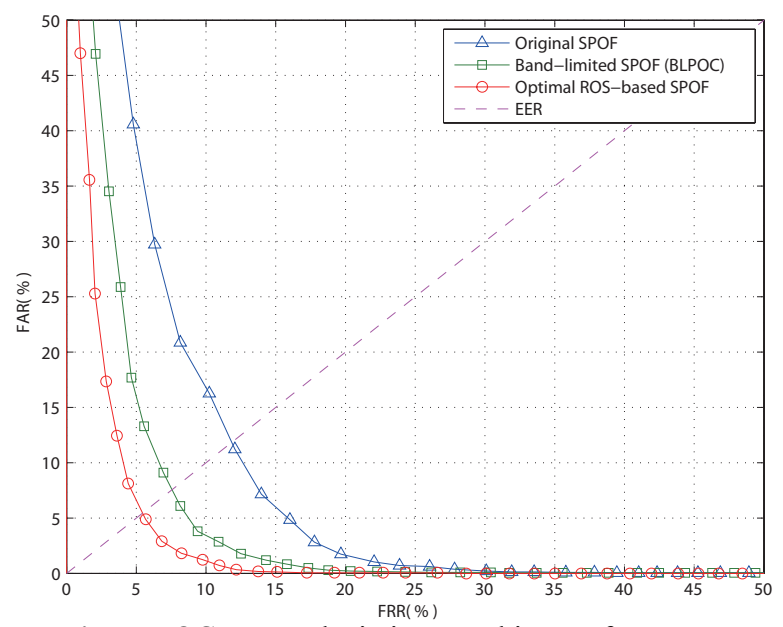

Fig. 5 ROC curves depicting matching performance.

gerprint verification. By means of theoretical and experimental analysis, the optimal ROS of SPOF was found to be the interior of the outer boundary of the frequency band corresponding to the fingerprint ridge lines. We also proposed the algorithm to determine the optimal ROS in practice.

Experiments have shown that the performance of SPOF with our optimal ROS is more efficient than that of BLSPOF (BLPOC).

\section{ACKNOWLEDGMENT}

This work is supported by research funds from NSFC No. 60572043 and the NKBRPC No.2004CB318005.

\section{REFERENCES}

[1] D. Maltoni, D. Maio, A. K. Jain and S. Prabhakar, Handbook of Fingerprint Recognition, Springer, New York, 2003.

[2] A.K. Jain, L. Hong and R. Bolle, "On-line Fingerprint Verification", IEEE Trans. Pattern Analysis and Machine Intelligence, 19(4): 302-314, 1997.

[3] A. M. Bazen, G.T.B. Verwaaijen, S. H. Gerez, L.P.J. Veelenturf and B.J.V.D. Zwaag, "A Correlation-Based Fingerprint Verification System", Proc. ProRISC2000 Workshop on Circuits, Systems and Signal Processing, pp.205-213, 2000.

[4] Q. Chen, M. Defrise, and F. Deconinck, "Symmetric PhaseOnly Matched Filtering of Fourier-Mellin Transforms for Image Registration and Recognition", IEEE Trans. Pattern Analysis and Machine Intelligence, 16(12): 1156-1168, 1994.

[5] K. Ito, H. Nakajima, K. Kobayashi, T. Aoki, and T. Higuchi, "A Fingerprint Matching Algorithm Using Phase-Only Correlation", IEICE Trans. Fundamentals, Vol. E87-A, No.3, pp. 682-691, 2004.

[6] B.V.K. V. Kumar, A. Mahalanobis, and R.D. Juday, Correlation Pattern Recognition, Cambridge University Press, UK, 2005.

[7] X. Zhan, Z. Sun, Y. Yin and Y. Chu, "Fingerprint Ridge Distance Estimation: Algorithms and the Performance", Proc. International Conference on Biometrics, pp. 294-301, 2006. 